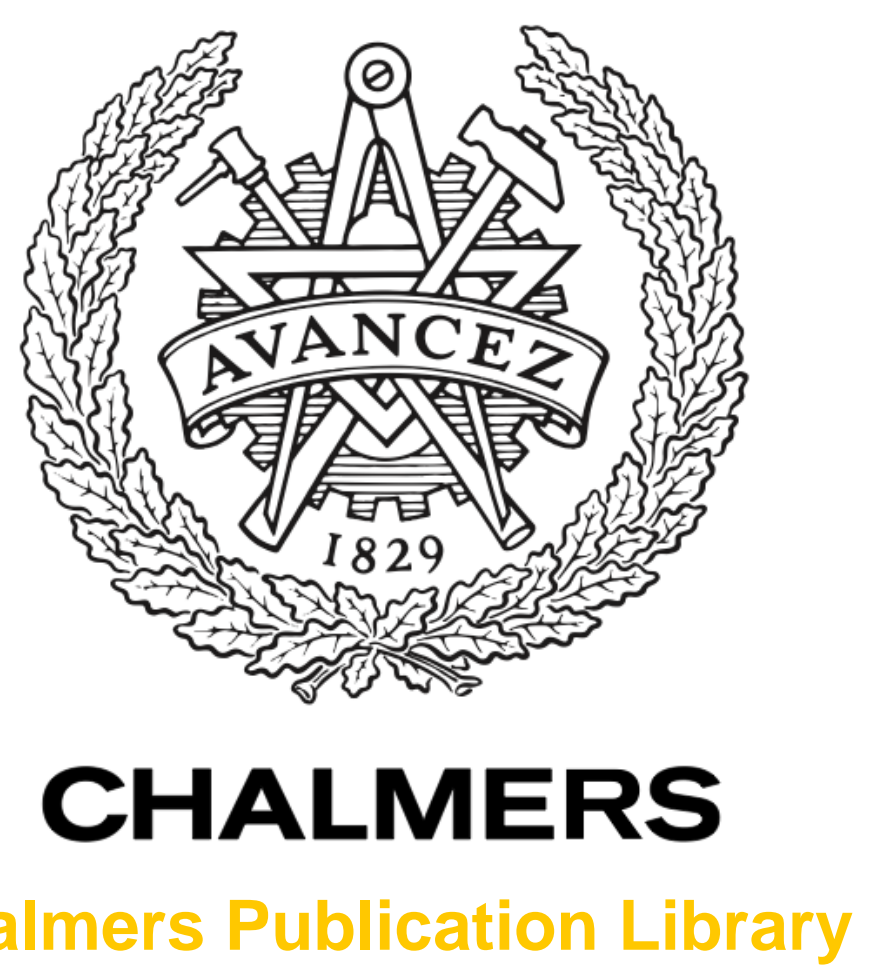

Challmers Publication Library

\title{
CSI feedback in correlated slow-fading channels
}

This document has been downloaded from Chalmers Publication Library (CPL). It is the author's version of a work that was accepted for publication in:

IEEE Communications Letters (ISSN: 1089-7798)

Citation for the published paper:

Makki, B. ; Eriksson, T. (2011) "CSI feedback in correlated slow-fading channels". IEEE

Communications Letters, vol. 15 pp. 1294 - 1297.

http://dx.doi.org/10.1109/LCOMM.2011.101011.11

Downloaded from: http://publications.lib.chalmers.se/publication/146302

Notice: Changes introduced as a result of publishing processes such as copy-editing and formatting may not be reflected in this document. For a definitive version of this work, please refer to the published source. Please note that access to the published version might require a subscription.

Chalmers Publication Library (CPL) offers the possibility of retrieving research publications produced at Chalmers University of Technology. It covers all types of publications: articles, dissertations, licentiate theses, masters theses, conference papers, reports etc. Since 2006 it is the official tool for Chalmers official publication statistics. To ensure that Chalmers research results are disseminated as widely as possible, an Open Access Policy has been adopted.

The CPL service is administrated and maintained by Chalmers Library. 


\title{
CSI feedback in correlated slow-fading channels
}

\author{
Behrooz Makki, Thomas Eriksson
}

\begin{abstract}
This letter studies the effect of quantized channel state information (CSI) feedback on the average rate of correlated channels. Demonstrating the general rate optimization problem, the results are obtained for both delayed and delay-free feedback conditions under different short- and long-term power allocation strategies. We also evaluate the effect of adaptive CSI quantization on the channel average rate. Analytical and numerical results show that exploiting the channel memory not only increases the forward channel data transmission efficiency but also can lead to dramatic feedback rate reduction.
\end{abstract}

Index Terms-Slow-fading correlated channel, CSI feedback, channel average rate, feedback compression.

\section{INTRODUCTION}

Due to the signaling overhead caused by reporting the channel state information (CSI), it is not feasible to provide the transmitter with perfect CSI in many applications. This is the main motivation for the present partial CSI feedback schemes, e.g., [1], [2], and this paper as well. In these methods, the transmitter is provided with as much as possible CSI via a minimum number of feedback bits. Further, among different research projects involving in this topic we can mention the 3rd Generation Partnership Project (3GPP) [3] in which partial CSI is one of the most important issues.

Implementing delay-free quantized CSI feedback, [2] investigated the channel average rate for uncorrelated slow-fading channels. Their results were later extended by us [1] where the system performance was studied in the presence of hybrid automatic repeat request feedback. However, realistic channels are often not memoryless. Simulations and practical measurements [4], [5] show high correlation between adjacent blocks, and exploiting the channel memory is expected to improve the channel data transmission efficiency. Therefore, in this paper we study the channel average rate in the case where there is correlation between successive channel realizations.

In comparison to previous works [1], [2], the new contributions of the paper are as follows: 1) For different power allocation strategies, the average rates are obtained under correlated channel conditions. 2) We investigate the effect of feedback delay on the achievable rates of the channel. 3) Theoretical bounds of feedback rate are obtained under different power allocation strategies and finally, 4) the results are extended to the case of adaptive CSI quantizers. Analytical and numerical results show that exploiting the channel memory not only increases the forward channel data transmission efficiency but also can lead to substantial feedback rate reduction. The results would be interesting for people involved in, e.g., 3GPP.

\section{SYSTEM MODEL}

Consider a communication channel in which the powerlimited input message $X$ multiplied by the fading random variable $h$ is summed with an iid complex Gaussian noise $Z: \mathcal{C N}\left(0, N_{0}\right)$ resulting in the output

$$
Y=h X+Z \text {. }
$$

We focus on correlated Markov slow-fading channels with $M$-steps-behind dependencies. That is, the channel gain $g=$ $|h|^{2}$ remains constant for a long time, generally determined by the channel coherence time, and then changes according to the fading probability density function (pdf) $f_{g^{k} \mid g^{k-1}, \ldots, g^{k-M}}\left(x_{0} \mid x_{1}, \ldots, x_{M}\right)$. It is assumed that there is perfect instantaneous knowledge about the channel gain at the receiver, which is an acceptable assumption under slow-fading conditions [1], [2]. Further, the feedback bits are supposed to be received by the transmitter error-free. Finally, with no loss of generality, we set the noise variance $N_{0}=1$.

Notations: In the sequel, $g^{k}=\left|h^{k}\right|^{2}$ represents the channel gain realization at time slot $k$, with the gain joint pdf $f_{g^{k}, \ldots, g^{k-M}}\left(x_{0}, \ldots, x_{M}\right)$. A quantization encoder

$$
Q^{k}=j, \text { if } g^{k} \in A_{j}=\left[\alpha_{j-1}, \alpha_{j}\right), \alpha_{0}=0, \alpha_{N}=\infty
$$

is implemented at the receiver where $N$ is the number of quantization regions (QRs), $\alpha_{j}$ 's denote the quantization boundaries and $A_{j}$ is the $j$-th QR. Moreover, the quantization decoder at the transmitter is represented by $I^{k}=j$, if $g^{k} \in A_{j}$. Further,

$$
\begin{aligned}
& \pi_{I^{k} \ldots I^{k-M}}=\operatorname{Pr}\left\{g^{k-m} \in A_{I^{k-m}}, m=0 \ldots M\right\} \\
& =\int_{A_{I^{k}}} \ldots \int_{A_{I^{k-M}}} f_{g^{k}, \ldots, g^{k-M}}\left(x_{0}, \ldots, x_{M}\right) \mathrm{d} x_{0} \ldots \mathrm{d} x_{M}
\end{aligned}
$$

denotes the probability that the successive channel realizations $g^{k}, g^{k-1}, \ldots, g^{k-M}$ fall into the $A_{I^{k}}, A_{I^{k-1}}, \ldots, A_{I^{k-M}}$ regions, respectively, and $T_{I^{k} \ldots I^{k-M}}$ is the transmission power in this case. Finally, $T$ is the average transmission power constraint.

\section{DELAY-FREE QUANTIZED CSI FEEDBACK}

Considering a delay-free quantized CSI feedback approach with $N$ QRs, the quantization encoder (2) is implemented at the receiver, and the transmitter is informed about the current quantization output. At the transmitter side, the quantization outputs are decoded by the decoding function

$$
I^{k}=j, \text { if } g^{k} \in A_{j}, j=1 \ldots N .
$$

The transmitter looks at the $M+1$ recent quantization indices $I^{k}, \ldots, I^{k-M}$, and selects a fixed gain $\hat{g}_{I^{k} \ldots I^{k-M}} \in A_{I^{k}}=$ $\left[\alpha_{I^{k}-1}, \alpha_{I^{k}}\right)$ accordingly. Then, data transmission is done at rate $R_{I^{k} \ldots I^{k-M}}=\log \left(1+\hat{g}_{I^{k} \ldots I^{k-M}} T_{I^{k} \ldots I^{k-M}}\right){ }^{1}$ If the instantaneous gain realization supports the rate, i.e., $g^{k} \geq \hat{g}_{I^{k} \ldots I^{k-M}}$, the data is successfully decoded, otherwise outage occurs. Hence, for every set of quantization indices $I^{k-m}, m=0 \ldots M$, the expected achievable rate of the channel is

$$
\begin{aligned}
& \bar{R}_{I^{k}} \ldots I^{k-M}= \\
& \operatorname{Pr}\left\{g^{k} \geq \hat{g}_{I^{k} \ldots I^{k-M}} \mid g^{k-m} \in A_{I^{k-m}}, m=0 \ldots M\right\} R_{I^{k} \ldots I^{k-M}} .
\end{aligned}
$$

Hence, using (3) and (5), the channel average rate is found as

\footnotetext{
${ }^{1}$ All results are presented in natural logarithm basis. Also, both the average and the feedback rates are presented in nats per channel use (npcu).
} 
$\bar{R}=\sum_{I^{k}=1}^{N} \ldots \sum_{I^{k-M}=1}^{N} \pi_{I^{k} \ldots I^{k-M}} \bar{R}_{I^{k} \ldots I^{k-M}}=$
$\sum_{I^{k}=1}^{N} \ldots \sum_{I^{k-M}=1} \eta_{I^{k} \ldots I^{k-M}} \log \left(1+\hat{g}_{I^{k} \ldots I^{k-M}} T_{I^{k} \ldots I^{k-M}}\right)$

where

$\eta_{I^{k} \ldots I^{k-M}} \doteq$

$\operatorname{Pr}\left\{\hat{g}_{I^{k} \ldots I^{k-M}} \leq g^{k}<\alpha_{I^{k}} \& g^{k-m} \in A_{I^{k-m}}, m=1 \ldots M\right\}=$ $\int_{\hat{g}_{I^{k} \ldots I^{k-M}}}^{\alpha_{I^{k}}} \int_{A_{I^{k-1}}} \ldots \int_{A_{I^{k-M}}} f_{g^{k}, \ldots, g^{k-M}}\left(x_{0}, \ldots, x_{M}\right) \mathrm{d} x_{0} \ldots \mathrm{d} x_{M}$

Equation (6) is based on the fact that, as the current gain realization is in the $I^{k}$-th region, the optimal considered gain $\hat{g}_{I^{k}} \ldots I^{k-M}$ must be within this region as well. Also, $\eta_{I^{k}} \ldots I^{k-M}$ is the probability that the rate $R_{I^{k} \ldots I^{k-M}}$ is decodable at the receiver. Finally, the average transmission power is obtained by

$$
\bar{T}=\sum_{I^{k}=1}^{N} \ldots \sum_{I^{k-M}=1}^{N} \pi_{I^{k} \ldots I^{k-M}} T_{I^{k} \ldots I^{k-M}} .
$$

In this perspective, the general power-limited rate optimization problem can be stated as

$$
\begin{gathered}
\bar{R}_{\max }=\max _{\forall T_{I^{k} \ldots I^{k-M}}, \hat{g}_{I^{k} \ldots I^{k-M}, \alpha_{j}}} \bar{R}\left(T_{I^{k} \ldots I^{k-M}}, \hat{g}_{I^{k} \ldots I^{k-M}}, \alpha_{j}\right) \\
\text { subject to } \bar{T} \leq T
\end{gathered}
$$

which, based on the considered power allocation strategy, can be solved numerically or analytically. Normally, there are two different interpretations of the power constraint. Short-term power allocation [1], [2] implies that $T_{I^{k} \ldots I^{k-M}}=T, \forall I^{k} \ldots I^{k-M}$. Under the more relaxed long-term power constraint, the transmitter can adapt the power based on channel conditions such that the average transmission power $\bar{T}$ does not exceed $T$. In this way, the optimal powers maximizing (6) can be found based on (6), (7) and a Lagrange multiplier approach, leading to the following water-filling equations

$$
T_{I^{k} \ldots I^{k-M}}=\left\lceil\frac{\eta_{I^{k} \ldots I^{k-M}}}{\lambda \pi_{I^{k} \ldots I^{k-M}}}-\frac{1}{\hat{g}_{I^{k} \ldots I^{k-M}}}\right\rceil^{+} \text {. }
$$

Here, $\lambda$ is the Lagrange multiplier constant obtained by (7). Based on (6)-(8), there are some interesting points such as:

- With long-term power allocation, the power is not wasted on weak channel realizations and the saved power is spent on strong channel conditions. That is, with limited power, no data is transmitted if the channel falls in low QRs and the power is preferably given to higher regions. This point also affects the optimal quantization boundaries, as the low regions with no power should be merged together.

- Setting $\hat{g}_{I^{k} \ldots I^{k-M}}=\hat{g}_{I^{k}} \forall I^{k-1} \ldots I^{k-M}$, which is a special case of the general average rate optimization problem, simplifies the results to the ones obtained under memoryless conditions [2]. Hence, as also emphasized in simulations, exploiting the channel memory leads to higher (or, in the worst case, equal) forward channel average rate.

\section{Minimum FEEDBACK RATE}

Based on the channel correlation properties, there are different practical methods such as differential coding [4] or sub-sampling [5] which can exploit the channel memory for feedback compression. However, it is always interesting to find the theoretical minimum feedback rate of the channel; considering the proposed correlated channel model $f_{g^{k} \mid g^{k-1}, \ldots, g^{k-M}}\left(x_{0} \mid x_{1}, \ldots, x_{M}\right)$, the channel theoretical minimum feedback rate is found as

$$
\begin{aligned}
& \bar{O}=H\left(I^{k} \mid I^{k-1}, \ldots, I^{k-M}\right)= \\
& \begin{array}{r}
\sum_{I^{k-1}=1}^{N} \ldots \sum_{I^{k-M}=1}^{N} \pi_{I^{k-1}} \ldots I^{k-M} \times \\
H\left(I^{k} \mid g^{k-m} \in A_{I^{k-m}}, m=1 \ldots M\right)
\end{array}
\end{aligned}
$$

where $H(U \mid V)$ is the conditional entropy of random variable $U$ given $V$ and $\pi_{I^{k-1} \ldots I^{k-M}}=\sum_{I^{k}=1}^{N} \pi_{I^{k} I^{k-1} \ldots I^{k-M}}$. Then, as conditioning reduces the entropy, it is obvious that using the channel memory can reduce the memoryless channel feedback rate obtained by $H\left(I^{k}\right)=-\sum_{I^{k}=1}^{N} \pi_{I^{k}} \log \left(\pi_{I^{k}}\right), \pi_{I^{k}}=$ $\sum_{I^{k-1}=1}^{N} \ldots \sum_{I^{k-M}=1}^{N} \pi_{I^{k} I^{k-1} \ldots I^{k-M}}$. Finally, from (9), the feedback rate reaches zero $\left(H\left(I^{k}\right)\right)$ in full (no) correlation conditions.

\section{D-STEPS DELAYED QUANTIZED CSI FEEDBACK}

Given that the transmitter is provided with $D$-steps delayed CSI feedback $I^{k-m}, m=D \ldots M, 1 \leq D \leq M$, it assumes the current gain realization $g^{k}$ to be $\hat{g}_{I^{k-D}} \ldots I^{k-M}$ which, in contrast to before, is not necessarily limited to any $\mathrm{QR}$. The data is sent with power $T_{I^{k-D}} \ldots I^{k-M}$ and rate $R_{I^{k-D}} \ldots I^{k-M}=$ $\log \left(1+\hat{g}_{I^{k-D} \ldots I^{k-M}} T_{I^{k-D}} \ldots I^{k-M}\right)$ which is received if $g^{k} \geq$ $\hat{g}_{I^{k-D}} \ldots I^{k-M}$. In this way, using the same procedure as before, the channel average rate is found as

$$
\bar{R}=\sum_{I^{k-D}=1}^{N} \ldots \sum_{I^{k-M}=1}^{N} \varphi_{I^{k-D} \ldots I^{k-M}} R_{I^{k-D} \ldots I^{k-M}}
$$

where we have

$$
\begin{aligned}
\varphi_{I^{k-D}} \ldots I^{k-M}= & \int_{\hat{g}_{I^{k-D} \ldots I^{k-M}}^{\infty}}^{\infty} \int_{-\infty}^{\infty} \ldots \int_{-\infty}^{\infty} \int_{A_{I^{k-D}}} \ldots \\
& \int_{A_{I^{k-M}}} f_{g^{k}, \ldots, g^{k-M}}\left(x_{0}, \ldots, x_{M}\right) \mathrm{d} x_{0} \ldots \mathrm{d} x_{M}
\end{aligned}
$$

Finally, using (3), the average input power is obtained by

$$
\bar{T}=\sum_{I^{k-D}=1}^{N} \ldots \sum_{I^{k-M}=1}^{N} \pi_{I^{k-D}} \ldots I^{k-M} T_{I^{k-D}} \ldots I^{k-M}
$$

which along with (10) change the rate optimization problem correspondingly.

\section{ADAPTIVE CHANNEL QUANTIZATION}

The forward channel average rate can be further increased by using adaptive quantizers [6]. This is particularly because adaptive quantizers can provide more accurate CSI at the transmitter by dynamic monitoring of the channel variations in correlated conditions. Here, we consider a scheme where, instead of one fixed encoder function, $N^{M}$ different functions

$$
\begin{aligned}
& Q_{\left\{I^{k-1} \ldots I^{k-M}\right\}}^{k}=j, \text { if } g^{k} \in A_{j,\left\{I^{k-1} \ldots I^{k-M}\right\}} \\
& A_{j,\left\{I^{k-1} \ldots I^{k-M}\right\}}=\left[\alpha_{j-1,\left\{I^{k-1} \ldots I^{k-M}\right\}}, \alpha_{j,\left\{I^{k-1} \ldots I^{k-M}\right\}}\right)
\end{aligned},
$$

each one associated with a specific set of previous quantization outputs $\left\{Q_{\left\{I^{k-2} \ldots I^{k-M-1}\right\}}^{k-1} \ldots Q_{\left\{I^{k-M-1} \ldots I^{k-2 M}\right\}}^{k-M}\right\}$, are implemented at the receiver. For each function, the quantization boundaries $\alpha_{j,\left\{I^{k-1} \ldots I^{k-M}\right\}}$ are dependent on the previous quantization outputs. Correspondingly, we consider $N$ fixed gain points $\hat{g}_{j,\left\{I^{k-1} \ldots I^{k-M}\right\}} \in A_{j,\left\{I^{k-1} \ldots I^{k-M}\right\}}$ and transmission powers $T_{j,\left\{I^{k-1} \ldots I^{k-M}\right\}}$ for each function. Finally, the decoder at the transmitter can be kept in synchronization with the encoders at the receiver, since both have access to the same history of indices.

Considering these new functions and replacing the optimization parameters by the new ones, the average rates are 
determined with the same procedure as before. Note that these new parameters are determined off-line at both transmitter and receiver, and so does not increase the feedback rate. Finally, as extending the results to delayed feedback case is straightforward, we do not discuss it further.

\section{Simulation RESUlts}

The results are obtained for Rayleigh-fading channels $f_{g^{k}}(g)=\frac{1}{\mu} e^{-\frac{g}{\mu}}, g \geq 0$ with 1-step-behind correlation modeled by $h^{k}=\beta h^{k-1}+\sqrt{1-\beta^{2}} \varepsilon, \varepsilon: \mathcal{C N}(0, \mu), g^{k}=\left|h^{k}\right|^{2}$.

Here, $\mu$ is the exponential pdf parameter and $\beta$ is the correlation factor demonstrating the two successive gain realizations dependencies. Under this model, the gain joint pdf is found as

$$
f_{g^{k}, g^{k-1}}(x, y)=\frac{1}{\left(1-\beta^{2}\right) \mu^{2}} e^{-\frac{x+y}{\left(1-\beta^{2}\right) \mu}} \Psi_{0}\left(\frac{2 \beta \sqrt{x y}}{\left(1-\beta^{2}\right) \mu}\right)
$$

where $\Psi_{0}($.$) is the zeroth-order modified Bessel function of$ the first kind [7]. Therefore, e.g., $\pi_{I^{k} I^{k-1}}$ and $\eta_{I^{k} I^{k-1}}$ can be determined based on the following integration procedure

$$
\begin{aligned}
& \int_{u}^{v} \int_{w}^{z} f_{g^{k}, g^{k-1}}(x, y) \mathrm{d} x \mathrm{~d} y \\
& \stackrel{(a)}{=} \int_{u}^{v} \frac{1}{\mu} e^{-\frac{x}{r}}\left(\int_{\sqrt{\frac{2 w}{r}}}^{\sqrt{\frac{2 z}{r}}} \theta e^{-\frac{\theta^{2}}{2}} \Psi_{0}(s \sqrt{x} \theta) \mathrm{d} \theta\right) \mathrm{d} x \\
& \stackrel{(b)}{=} \int_{u}^{v} \frac{1}{\mu} e^{-\frac{x}{\mu}}\left\{\xi\left(s \sqrt{x}, \sqrt{\frac{2 w}{r}}\right)-\xi\left(s \sqrt{x}, \sqrt{\frac{2 z}{r}}\right)\right\} \mathrm{d} x \\
& \stackrel{(c)}{=} e^{-\frac{w}{\mu}}\left\{\xi\left(\sqrt{\frac{2 w}{r}} \beta, \sqrt{\frac{2 u}{r}}\right)-\xi\left(\sqrt{\frac{2 w}{r}} \beta, \sqrt{\frac{2 v}{r}}\right)\right\} \\
& -e^{-\frac{z}{\mu}}\left\{\xi\left(\sqrt{\frac{2 z}{r}} \beta, \sqrt{\frac{2 u}{r}}\right)-\xi\left(\sqrt{\frac{2 z}{r}} \beta, \sqrt{\frac{2 v}{r}}\right)\right\} \\
& +e^{-\frac{v}{\mu}} \xi\left(\sqrt{\frac{2 w}{r}}, \sqrt{\frac{2 v}{r}} \beta\right)-e^{-\frac{u}{\mu}} \xi\left(\sqrt{\frac{2 w}{r}}, \sqrt{\frac{2 u}{r}} \beta\right) \\
& -e^{-\frac{v}{\mu}} \xi\left(\sqrt{\frac{2 z}{r}}, \sqrt{\frac{2 v}{r}} \beta\right)+e^{-\frac{u}{\mu}} \xi\left(\sqrt{\frac{2 z}{r}}, \sqrt{\frac{2 u}{r}} \beta\right)
\end{aligned}
$$

Here, (a) is obtained by defining $r \doteq\left(1-\beta^{2}\right) \mu, s \doteq$ $\sqrt{2 / r} \beta$ and using variable transform $\theta=\sqrt{2 y / r}$. Then, (b) is directly obtained from the definition of the Marcum Qfunction $\xi(x, y)=\int_{y}^{\infty} t e^{-\frac{t^{2}+x^{2}}{2}} \Psi_{0}(x t) \mathrm{d} t$ and (c) is derived by $\xi(x, y)=1+e^{-\left(x^{2}+y^{2}\right) / 2} \Psi_{0}(x y)-\xi(y, x)$, using variable transform $t=\sqrt{x}$, partial integration and some calculations.

Considering $\mu=1, \beta=0.8$ and $N=2$ QRs (if not otherwise mentioned $)^{2}$, Fig.1a shows the channel average rate under different data transmission strategies. Also, the results obtained under no knowledge, i.e., (7) in [2], and full knowledge assumptions have been plotted as two lower and upper bounds, respectively. Moreover, considering fixed quantizers and different input powers, Fig.1b verifies the effect of channel memory on the channel feedback rate for different power allocations. Finally, utilizing fixed equal-probability quantizers, Fig.2 shows the average rate versus the feedback rate for different number of QRs.

\section{DISCUSSIONS AND CONCLUSION}

This report studies the average rate of correlated slowfading channels in the presence of quantized CSI feedback. The results show that: 1) utilizing fixed quantizers, there is a small average rate gain when exploiting the channel memory. However, in harmony with practice [4], [5], substantial feedback compression is obtained even with fixed quantizers (Fig.2).

\footnotetext{
${ }^{2}$ While not shown here, the simulations have been run for different parameter settings with similar qualitative results.
}
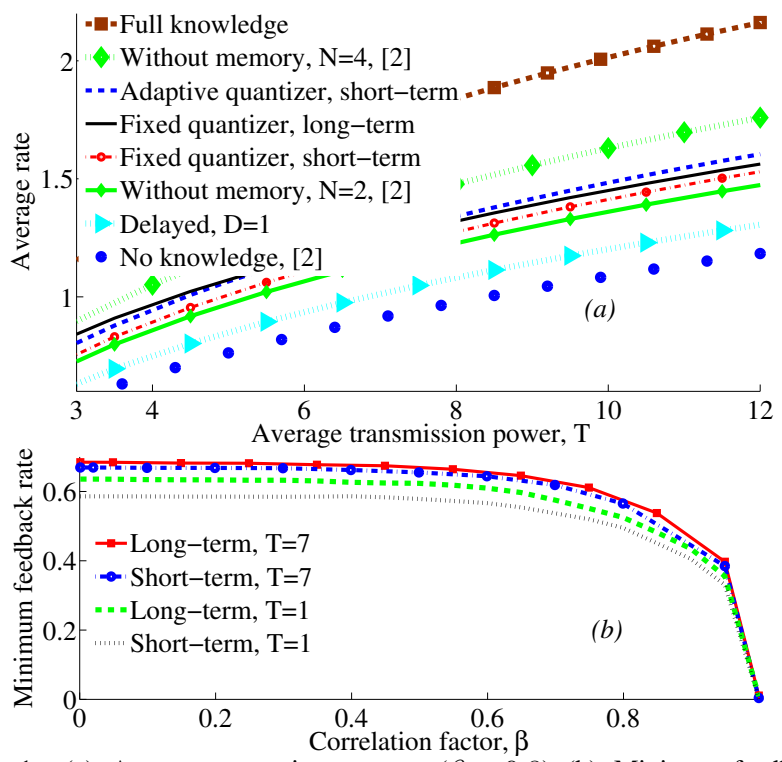

Figure 1. (a): Average rate vs input power, $(\beta=0.8)$. (b): Minimum feedback rate vs correlation factor $\beta,(T=1 \& 7) . \mu=1, N=2$ if not mentioned.

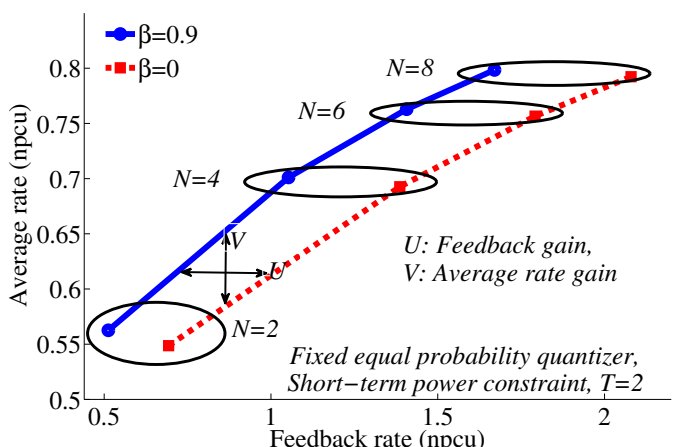

Figure 2. Average rate vs feedback rate, short-term power constraint $T=2$.

2) Further, adaptive quantization considerably improves the average rate. For instance, in Rayleigh-fading channels with 1 -step-behind dependency $(\beta=0.8)$, (almost) half the gain obtained in the forward link by $N=4 \mathrm{QRs}$ is achieved by utilizing adaptive quantizers with $N=2$ QRs (Fig.1a). 3) long-term power allocation increases both the forward channel average rate and the feedback rate. Therefore, its optimality is under question when both metrics are considered as optimization criterion. However, these effects diminish in high SNR regimes (Fig.1b). 4) Depending on the channel memory, considerable rate increment can be achieved even with delayed CSI feedback (Fig.1a). 5) The feedback rate decreases as the transmission power (the channel correlation) reduces (increases) (Fig.1b).

\section{REFERENCES}

[1] B. Makki, et. al, "On the average rate of quasi-static fading channels with ARQ and CSI feedback," Commun. Lett., vol. 14, no. 9, pp. 806-808, 2010.

[2] T. T. Kim and M. Skoglund, "On the expected rate of slowly fading channels with quantized side information," IEEE Trans. on Commun., vol. 55, no. 4, pp. 820-829, April 2007.

[3] www.3gpp.org.

[4] R1-062772, "Compressed CQI reporting scheme," NEC, RAN WG1 meeting 46, Seoul, Republic of Korea, Oct. 2006.

[5] T. Eriksson and T. Ottosson, "Compression of feedback in adaptive OFDMbased systems using scheduling," Commun. Lett., vol. 11, no. 11, pp. 859861, Nov. 2007.

[6] K. Sayood, Introduction to Data Compression. Morgan Kaufmann Publishers, 3rd ed., 2006.

[7] C. Tellambura, et. al, "Generation of bivariate rayleigh and nakagami-m fading envelopes," Commun. Lett., vol. 4, no. 5, pp. 170-172, May 2000. 